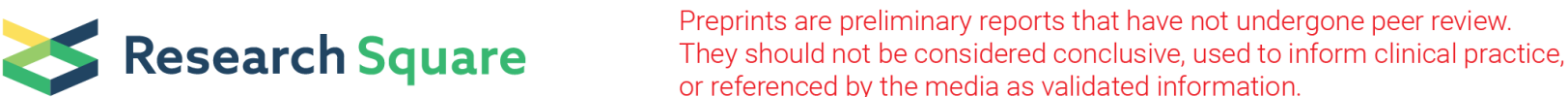

\section{A Practical Strategy of Controlled Monopolar Cautery With/Without Oversewing for Staple Line Bleeding in Bariatric Surgery: A Single Center Experience}

\author{
Fang-Chin Hsu \\ Tri-Service General Hospital \\ Hai-Ning Hsu \\ Tri-Service General Hospital \\ Hsin-Mei Pan \\ Tri-Service General Hospital \\ Wan-Ting Hung \\ Tri-Service General Hospital \\ Guo-Shiou Liao \\ Tri-Service General Hospital \\ Teng-Wei Chen \\ Tri-Service General Hospital \\ De-Chuan Chan \\ Tri-Service General Hospital \\ Kuo-Feng Hsu ( $\nabla$ hsukf97@ndmctsgh.edu.tw ) \\ Tri-Service General Hospital
}

\section{Research Article}

Keywords: monopolar cautery, oversewing, staple line bleeding, bariatric surgery.

Posted Date: September 9th, 2021

DOI: https://doi.org/10.21203/rs.3.rs-880492/v1

License: (c) (i) This work is licensed under a Creative Commons Attribution 4.0 International License. Read Full License 


\section{Abstract \\ Introduction}

In laparoscopic bariatric surgery, stapling devices are essential for creating a gastric tube or gastric pouch. Staple line bleeding (SLB) is an important issue, but the effect of monopolar cautery for SLB control is unclear. This study aimed to evaluate the outcomes of controlled monopolar cautery with/without oversewing for SLB control during bariatric surgery.

\section{Materials and Methods}

From January 2019 to May 2021, 66 patients underwent bariatric surgery with controlled monopolar cautery with/without oversewing for SLB control. Patient demographics and intraoperative and postoperative outcomes were analyzed.

\section{Results}

This study enrolled 35 women and 31 men (mean age: 38.1 years; mean body mass index: $39.8 \mathrm{~kg} / \mathrm{m}^{2}$ ). All patients had varying degrees of SLB. Controlled monopolar cautery was applied to 63 patients with $\leq 6$ bleeding sites. Only two patients needed further partial oversewing of the bleeding sites after controlled monopolar cautery failed to achieve hemostasis. Initially, three patients had more than six bleeding sites, and thus complete oversewing was performed to achieve successful hemostasis. There was no intraabdominal or gastrointestinal bleeding. There were one gastric stricture, one anastomotic leakage, and one staple line leakage.

\section{Conclusions}

The strategy of controlled monopolar cautery with/without oversewing is a simple, practical, inexpensive, and safe method for SLB control during bariatric surgery.

\section{Key Points}

- Staple line bleeding is an important issue during laparoscopic bariatric surgery.

- We propose a concept of controlled monopolar cautery for staple line bleeding control, and give a precise instruction for surgeons to perform it.

- The strategy of controlled monopolar cautery with/without oversewing is a simple, effective, and safe approach for staple line bleeding control during bariatric surgery.

\section{Introduction}


Obesity is a serious, costly issue with increasing prevalence worldwide. Bariatric surgery is more effective for managing obesity than intensive medical therapy alone [1, 2]. Numerous bariatric surgeries have been well-performed for decades. The American Society for Metabolic and Bariatric Surgery considers the Roux-en-Y gastric bypass (RYGB) the gold standard. However, complications such as potential malnutrition, iron deficiency anemia, marginal ulcers, and increased risk of gastroesophageal reflux disease (GERD) could make this option less attractive $[3,4]$.

Sleeve gastrectomy (SG) has become the predominant choice of bariatric surgery due to its simplicity and effectiveness of weight loss[5]. However, weight regain and worse control of comorbidities than RYGB are always concerns [6]. Hence, the sleeve plus technique combines foregut exclusion with SG. It has both restrictive and malabsorptive effects, with reportedly better weight loss and comorbidity control [7].

No matter which laparoscopic bariatric surgery (i.e., SG, sleeve plus, or RYGB) requires the use of stapling devices to create a gastric tube or gastric pouch. The complication of staple line bleeding (SLB) remains an important issue perioperatively and postoperatively. There are various SLB control methods, such as cauterization, oversewing, tissue sealant, and buttress material, but optimal management remains controversial $[8,9]$. This study aimed to investigate our proposed strategy of controlled monopolar cautery with/without oversewing for SLB control during bariatric surgery.

\section{Materials And Methods}

We conducted a retrospective cohort study using our prospective database. We identified 66 patients who underwent bariatric surgery from January 2019 to May 2021 and grouped them according to surgery type: $S G(n=37)$, sleeve plus $(n=24)$, and gastric bypass surgery $(n=5)$. The sleeve plus procedures included the following: (1) SG with proximal jejunal bypass, (2) SG with duodenal-jejunal bypass, (3) sleeve jejunal bypass, and (4) sleeve ileal bypass. The gastric bypass procedures included RYGB and mini-gastric bypass/one anastomosis gastric bypass. The study was approved by Ethical Committee of Tri-service general hospital (No.40728). All procedures performed in studies involving human participants complied with the ethical standards of the institutional and/or national research committee and the 1964 Helsinki declaration and its later amendments or comparable ethical standards. All individual participants provided written informed consent. We included patients aged $20-65$ years and with $\mathrm{BMl}>37.5 \mathrm{~kg} / \mathrm{m}^{2}$ or $\mathrm{BMl}>32.5 \mathrm{~kg} / \mathrm{m}^{2}$ with comorbidities. We excluded patients who underwent open bariatric surgery and those with cirrhosis. A team consisting of a surgeon, endocrinologist, dietician, and psychiatrist evaluated all patients before bariatric surgery.

All patients underwent laparoscopic procedures. The patient was put in the supine position under general anesthesia. Then, 3-5 trocars were placed. A gastric tube or a gastric pouch was created with linear staples (Medtronic Endo-GIA Universal - $60 \mathrm{~mm}$-blue/purple/green load), and the anastomotic openings of gastrojejunostomy or gastroileostomy in the bypass procedure were closed using $3 / 0$ polypropylene sutures. For each case, we meticulously examined the staple line after gastric stapling and counted the 
bleeding sites. In patients with $\leq 6$ bleeding sites, controlled monopolar cautery was tried initially to achieve hemostasis. "Controlled monopolar cautery" is defined as using a coagulation mode level of 20 watts, each attempt lasting not more than 1 second with a maximum of three attempts. If hemostasis could not be achieved via controlled monopolar cautery, we only oversewed the bleeding site (Fig. 1). In patients with more than six initial bleeding sites, we directly performed complete oversewing. Table 1 shows patient characteristics. The intraoperative and postoperative data were analyzed as well (Table 2). 
Table 1

Characteristics of the patients undergoing bariatric surgery

\begin{tabular}{|c|c|}
\hline Variables & Patients $(n=66)$ \\
\hline Age, year & $38.1 \pm 11.7$ \\
\hline Gender (male/female) & $31 / 35$ \\
\hline Body weight, kg & $112 \pm 21.8$ \\
\hline Body height, $\mathrm{m}$ & $1.67 \pm 0.091$ \\
\hline Body mass index, $\mathrm{kg} / \mathrm{m}^{2}$ & $39.8 \pm 5.6$ \\
\hline \multicolumn{2}{|l|}{ Operations } \\
\hline Sleeve gastrectomy & $37(56.1 \%)$ \\
\hline Sleeve plus procedure & $24(36.4 \%)$ \\
\hline Gastric bypass & $5(7.5 \%)$ \\
\hline \multicolumn{2}{|l|}{ Concomitant surgeries } \\
\hline Cholecystectomy & $10(15.2 \%)$ \\
\hline Hiatal hernia repair & $7(10.6 \%)$ \\
\hline Revisional bariatric surgery & $6(9.1 \%)$ \\
\hline From SG to RYGB & 1 \\
\hline From RYGB to gastric pouch resizing and distalization of roux limb & 1 \\
\hline From SG to re-SG & 1 \\
\hline From gastric clipping to SG & 3 \\
\hline \multicolumn{2}{|l|}{ ASA classification } \\
\hline Class I & $12(18.2 \%)$ \\
\hline Class II & $44(66.7 \%)$ \\
\hline Class III & $10(15.2 \%)$ \\
\hline \multicolumn{2}{|l|}{ Comorbidities } \\
\hline T2DM & $21(31.8 \%)$ \\
\hline \multicolumn{2}{|l|}{ Data are presented as mean \pm standard deviation, $n(\%)$; ASA: } \\
\hline \multicolumn{2}{|l|}{ American Society of Anesthesiologists; T2DM: type 2 diabetes mellitus; } \\
\hline CCl: Charlson comorbidity index; INR: international normalized ratio & \\
\hline
\end{tabular}




\begin{tabular}{|ll|}
\hline Variables & Patients $(\mathbf{n}=\mathbf{6 6})$ \\
\hline Hypertension & $59(89.4 \%)$ \\
\hline Hyperlipidemia & $53(80.3 \%)$ \\
\hline Moderate/Severe obstructive sleep apnea & $13(19.7 \%)$ \\
\hline CCI & $1.14 \pm 0.88$ \\
\hline INR & $0.99 \pm 0.09$ \\
\hline Data are presented as mean \pm standard deviation, $n(\%)$; ASA: & \\
\hline American Society of Anesthesiologists; T2DM: type 2 diabetes mellitus; \\
\hline CCl: Charlson comorbidity index; INR: international normalized ratio \\
\hline
\end{tabular}

Table 2. Intraoperative and postoperative data of the patients undergoing bariatric surgery 


\begin{tabular}{ll} 
Variables & Patients ( $\mathrm{n}=66)$ \\
\hline Number of staples for stomach & $5.17 \pm 0.97$ \\
\hline Number of bleeders over staple line & $3.23 \pm 1.80$ \\
\hline Control for staple line bleeding (case) & \\
\hline Monopolar cautery only & 61 \\
\hline Monopolar cautery+ oversewing & 2 \\
\hline Oversewing & 3 \\
\hline Operation time, minute & $154.4 \pm 39.7$ \\
\hline Blood loss, ml & $14.3 \pm 13.7$ \\
\hline Drain placement (present) & $61(92.4 \%)$ \\
\hline Postoperative complications & \\
\hline Intra-abdominal bleeding & 0 \\
\hline Gastro-intestinal bleeding & 0 \\
\hline Stricture & 1 \\
\hline Anastomotic leakage & 1 \\
\hline Staple line leakage & 1 \\
\hline Wound infection & 0 \\
\hline Postoperative hospital stay (day) & $5.7 \pm 7.8$ \\
\hline
\end{tabular}

Data are presented as mean \pm standard deviation, $\mathrm{n}(\%)$

\section{Results}

Out of 66 patients, 37 underwent SG, 24 underwent sleeve plus, and five underwent RYGB. Regarding patient demographics, 35 patients (53\%) were female. The mean age and BMI were 38.1 years and 39.8 $\mathrm{kg} / \mathrm{m}^{2}$, respectively. Regarding comorbidities, 21 patients had type 2 diabetes mellitus (T2DM), 59 had hypertension, 53 had hyperlipidemia, and 13 had moderate to severe obstructive sleep apnea. Moreover, 17 patients underwent concomitant surgery, including ten cholecystectomies due to gallstones and seven hiatal hernia repairs. On the other hand, six patients underwent revisional bariatric surgery. Other baseline characteristics are summarized in Table 1.

All surgeries were successful without perioperative complications or conversion to open surgery. We used a mean of $5.17 \pm 0.97$ staples for stomach resection, with a mean of $3.23 \pm 1.80$ bleeders noted over the staple line (Table 2). All patients had varying degrees of SLB; 63 patients had $\leq 6$ bleeding sites, whereas 
only three patients had more than six bleeding sites. Patients with $\leq 6$ bleeding sites received controlled monopolar cautery. Hemostasis was successful in 61 patients, and only two patients failed to achieve hemostasis. For cases of failed hemostasis, partial oversewing of the bleeding sites was performed, and bleeding was controlled successfully after this. There were three patients with more than six initial bleeding sites; they underwent complete oversewing for successful hemostasis (Fig. 2).

The overall postoperative complication rate in this study was 4.5\% (3 events). One case was a gastric stricture in a 38-year-old female with persistent nausea and vomiting after SG. Two attempts at balloon dilatation of the stricture were unsuccessful. Thus, the patients underwent revisional RYGB. The second case is a 30-year-old female smoker with systemic lupus erythematosus who underwent single anastomosis sleeve jejunal bypass. She presented with acute abdomen seven days after discharge and was sent to the emergency department. Diagnostic laparoscopy revealed mild leakage over the gastrojejunal anastomosis; the patient received a primary suture and drainage for this leakage. The third case is a 28-year-old male who underwent concomitant hiatal hernia repair with laparoscopic SG. A staple line leakage over the esophagogastric junction (EGJ) was noted after surgery. Upon reviewing the surgical video, we found no monopolar cautery applied over the EGJ. We considered this due to a thermal injury caused by the LigaSure (Medtronic) during the hiatal hernia repair. Overall, these complications seem to be unrelated to the monopolar cautery procedure itself.

\section{Discussion}

Since laparoscopic SG and bypass surgery are commonly performed types of bariatric surgery, different techniques reduce staple line-related complications such as SLB and leakage. However, there is no consensus regarding the best technique. Several systematic reviews of staple line leakage after SG, comparing different reinforcement techniques, found a similar result. The leakage rate was significantly lower when using the absorbable polymer membrane (APM) staple line reinforcement than oversewing, fibrin sealant, bovine pericardial strips (BPS), and no reinforcement[10, 11]. However, using the APM, BPS, or other expensive commercial products may be a financial burden for patients [12].

As for SLB control, monopolar cautery is not always recommended because it may weaken the staple line, although it is a simple and rapid method for reaching hemostasis. However, Gundogan et al. recently investigated the efficacy of monopolar cautery versus clips for SLB control in RYGB [13]. Sağlam et al. used only monopolar cautery for SLB control in 187 patients undergoing SG [14]. Both studies found monopolar cautery to be a safe, effective, and inexpensive approach for SLB control. However, they did not describe the number and duration of attempts while using monopolar cautery; this technique might be difficult to apply in clinics. In this study, the controlled monopolar cautery was defined precisely in terms of duration and frequency. Postoperative outcomes revealed no intraabdominal or gastrointestinal bleeding. The only staple line leakage was over the EGJ, and this was considered a thermal injury due to the LigaSure during a concomitant hiatal hernia repair. 
Postoperative gastric stricture is a rare but serious complication following bariatric surgery. The routine complete oversewing of the staple line may prevent SLB, but this is time-consuming and must be performed by experienced surgeons to avoid gastric stricture [15]. A study conducted by Fort et al. compared complete and partial oversewing of the staple line [16]. They found that operative time was significantly longer, and the need for revision surgery was significantly higher in the complete oversewing group. In this study, we only performed complete oversewing in patients with more than six bleeding sites of the staple line. Partial oversewing was applied to the bleeding site in patients with failed hemostasis by monopolar cautery. In our opinion, partial oversewing may help overcome the shortcomings of complete oversewing and achieve satisfactory hemostasis. We noted a case of gastric stricture after SG in our study, but oversewing was not performed in this case. Upon reviewing the surgical video of this patient, the stricture appeared to be due to excessive traction of the stomach during gastric stapling.

In conclusion, SLB is a crucial issue in laparoscopic bariatric surgery, involving gastric stapling procedure. There are various SLB control methods. In this study, we found controlled monopolar cautery to be a safe, effective, and easy method to achieve hemostasis in cases of SLB. In cases when this technique failed, partial oversewing was applied to overcome the shortcomings of complete oversewing and achieve satisfactory hemostasis. Therefore, our proposed strategy of controlled monopolar cautery with/without oversewing can be a feasible option for SLB control.

\section{Declarations}

\section{Disclosure:}

Dr. Fang-Chin Hsu, Hai-Ning Hsu, Hsin-Mei Pan, Wan-Ting Hung, Guo-Shiou Liao Teng-Wei Chen, DeChuan Chan, Kuo-Feng Hsu have no conflicts of interest.

\section{References}

1. Schauer, P. R. et al. Bariatric Surgery versus Intensive Medical Therapy for Diabetes - 5-Year Outcomes. The New England journal of medicine, 376, 641-651 (2017).

2. Lee, J. H., Nguyen, Q. N. \& Le, Q. A. Comparative effectiveness of 3 bariatric surgery procedures: Roux-en-Y gastric bypass, laparoscopic adjustable gastric band, and sleeve gastrectomy. Surgery for obesity and related diseases: official journal of the American Society for Bariatric Surgery, 12, 9971002 (2016).

3. Bal, B. S., Finelli, F. C., Shope, T. R. \& Koch, T. R. Nutritional deficiencies after bariatric surgery. Nature reviews Endocrinology, 8, 544-556 (2012).

4. Tack, J. \& Deloose, E. Complications of bariatric surgery: dumping syndrome, reflux and vitamin deficiencies. Best practice \& research Clinical gastroenterology, 28, 741-749 (2014).

5. Angrisani, L. et al. IFSO Worldwide Survey 2016: Primary, Endoluminal, and Revisional Procedures. Obes. Surg, 28, 3783-3794 (2018). 
6. Velapati, S. R. et al. Weight Regain After Bariatric Surgery: Prevalence, Etiology, and Treatment. Current nutrition reports, 7, 329-334 (2018).

7. Huang, C-K., Liu, C-C., Hsin, M-C., Chen, Y-C-J-A-L. \& Surgery, E. (2017) Sleeve and sleeve plus. 20172

8. Mercier, G. et al. Surgical glue in laparoscopic sleeve gastrectomy: An initial experience and costeffectiveness analysis. J Eval Clin Pract, 23, 614-619 (2017).

9. Albanopoulos, K. et al. Reinforcing the staple line during laparoscopic sleeve gastrectomy: prospective randomized clinical study comparing two different techniques. Preliminary results. Obes Surg, 22, 42-46 (2012).

10. Gagner, M. \& Buchwald, J. N. Comparison of laparoscopic sleeve gastrectomy leak rates in four staple-line reinforcement options: a systematic review. Surgery for obesity and related diseases: official journal of the American Society for Bariatric Surgery, 10, 713-723 (2014).

11. Gagner, M. \& Kemmeter, P. Comparison of laparoscopic sleeve gastrectomy leak rates in five stapleline reinforcement options: a systematic review. Surgical endoscopy, 34, 396-407 (2020).

12. Consten, E. C., Gagner, M., Pomp, A. \& Inabnet, W. B. Decreased bleeding after laparoscopic sleeve gastrectomy with or without duodenal switch for morbid obesity using a stapled buttressed absorbable polymer membrane. Obes Surg, 14, 1360-1366 (2004).

13. Gundogan, E. et al. Randomized controlled trial of monopolar cautery versus clips for staple line bleeding control in Roux-en-Y gastric bypass. International journal of surgery (London, England), 58, 52-56 (2018).

14. Sağlam, K. et al. (2019) Staple line bleeding control with monopolar cautery in laparoscopic sleeve gastrectomy. 26:181-184

15. Taha, O., Abdelaal, M., Talaat, M. \& Abozeid, M. A Randomized Comparison Between Staple-Line Oversewing Versus No Reinforcement During Laparoscopic Vertical Sleeve Gastrectomy. Obes Surg, 28, 218-225 (2018).

16. Fort, J. M. et al. (2020) Management of the staple line in laparoscopic sleeve gastrectomy: comparison of three different reinforcement techniques. Surgical endoscopy

\section{Figures}




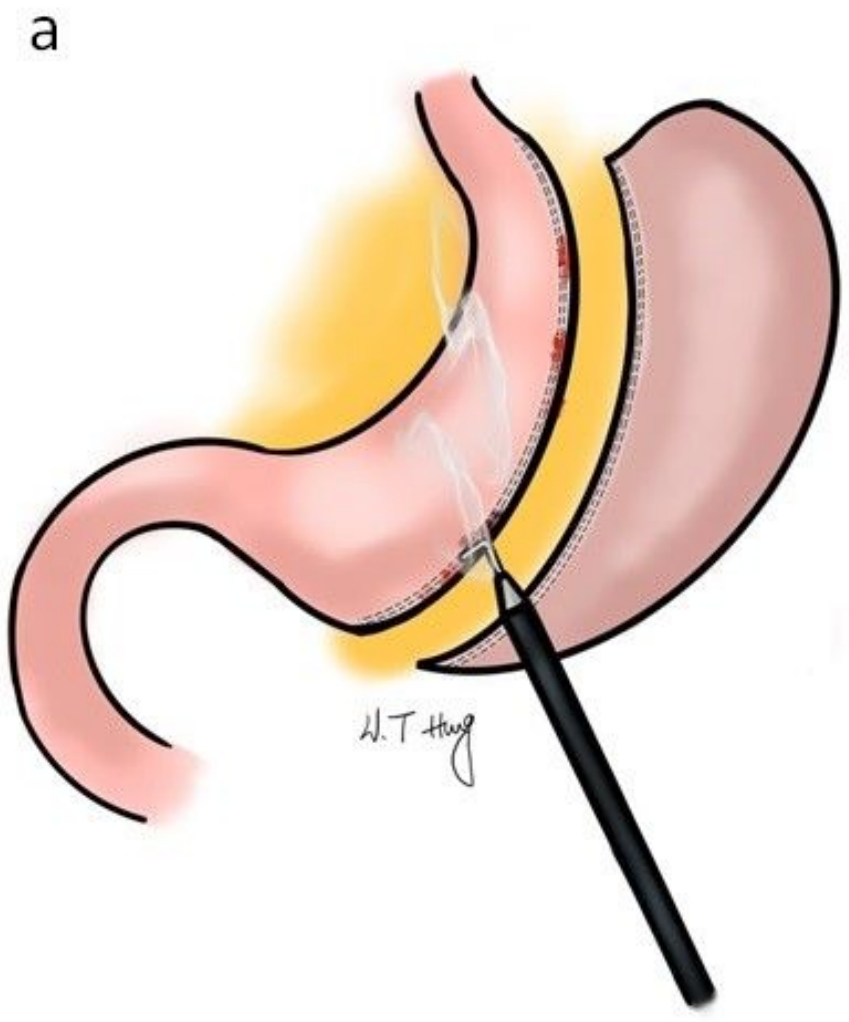

b

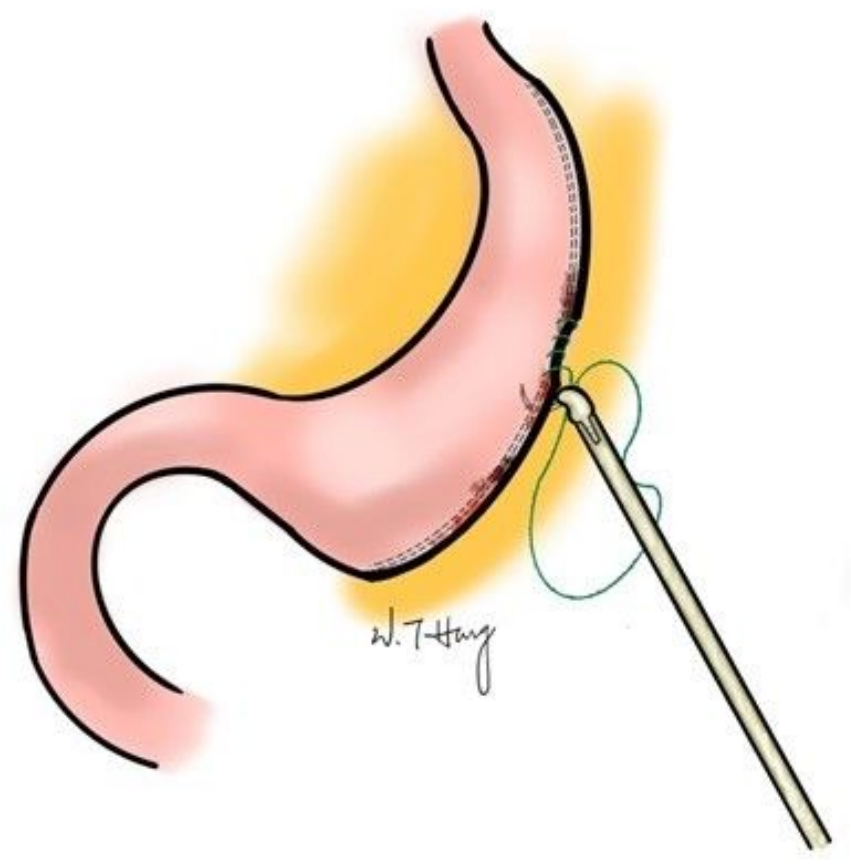

Figure 1

SLB control methods. (a) In patients with $\leq 6$ bleeding sites, controlled monopolar cautery was tried initially to achieve hemostasis. (b) In cases where controlled monopolar cautery failed to achieve hemostasis, we only performed oversewing on the bleeding site.

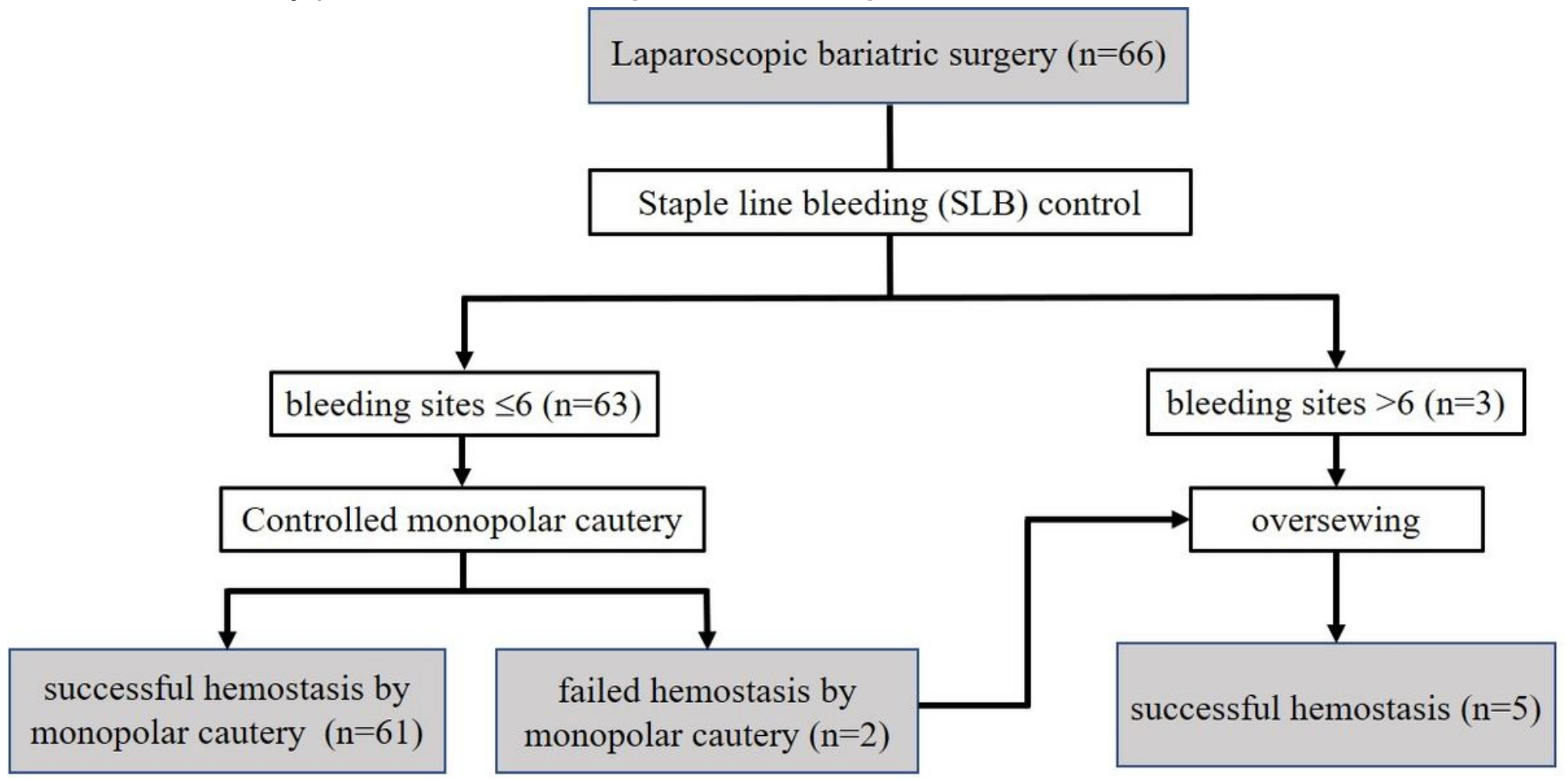




\section{Figure 2}

The flowchart of SLB control. All the patients had varying degrees of SLB. In 63 patients with $\leq 6$ bleeding sites, controlled monopolar cautery were tried initially. However, two patients had failed cauterization and thus needed partial oversewing. For the three patients with more than six bleeding sites, complete oversewing was done. Hemostasis was successful in all patients. 